\title{
Magnetic Technique for Nondestructive Evaluation of Residual Stresses
}

\author{
B. A. Belyaev, A. A. Leksikov, S. G. Ovchinnikov, I. Kraus, A. S. Parshin
}

A technique has been designed for measuring planar components of stray fields from ferromagnetic samples placed in a constant magnetizing field. The technique is based on recording the field of magnetization reversal of a thin magnetic film with the small coercive force being the sensor device of a microwave detector. The possibility of measuring the deformation inhomogeneities caused by mechanical treatment when manufacturing products from ferromagnetic materials is demonstrated. The results of the magnetic measurements agree with the data from $X$-ray diffraction analysis.

Keywords: ferromagnetic resonance, thin magnetic films, microvawe scanning spectrometer, residual stress

\section{Introduction}

As is known, during mechanical treatment of materials here arise elastic and plastic deformations, which may have an essential influence on the lifetime of products. In particular, specially created deformations on the surfaces of metal details increase their strength, operational reliability and lifetime [1]. Therefore the development of a technique and apparatus for nondestructive evaluation of deformations [2] is an important problem at the present time. Measurements of the distribution of the quantity of near-surface deformations on the area of details, and also the elastic stresses existing in them, are of great importance, as they significantly simplify the search for optimum conditions of treatment for each specific detail.

This paper shows in principle how the inhomogeneous elastic stresses in a sample of ferromagnetic material can be measured. The measurement technique is based on recording the inhomogeneities of stray fields near to a surface of a sample placed in a homogeneous magnetic field $H_{0}$, the quantity of which is much lower than the field of magnetic saturation.

The stray fields are determined by the magnetic domain structure on each local part of the sample, which depends on a set of factors including elastic stresses. In particular, elastic stresses, are known [3] to have a dramatic effect on the magnetic anisotropy of a material. Therefore the equilibrium orientation of the magnetic moments in domains on any local part of an investigated sample depends on the quantity and direction of the elastic stresses on this part.

\section{Measurement technique}

The stray fields are measured with the help of an original microwave detector [4] whose sensing element is a thin magnetic film (TMF) having uniaxial magnetic anisotropy. The detector (Fig. 1) functions on the basis of the measuring head of a scanning automated spectrometer of ferromagnetic resonance [5]. The transistor microwave generator and the detector are placed in the nonmagnetic metal body. The driver circuit of the generator is a microstrip resonator (MSR). The measuring hole is etched in the screen of the MSR. The measuring hole is the local source of the microwave magnetic field. This hole with a diameter of $\sim 1 \mathrm{~mm}$ is enclosed by a magnetic film in such a way that the axis of easy magnetization of the TMF is orthogonal to the direction of

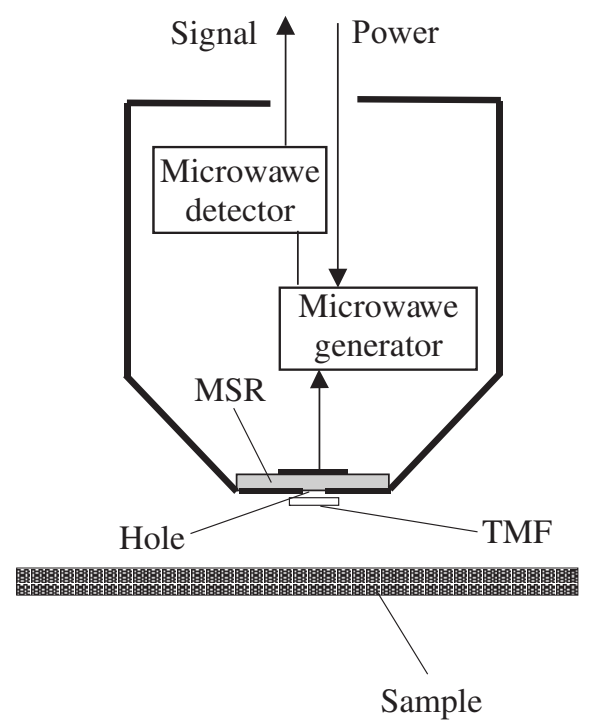

Fig. 1.: Layout of MW detector of magnetic field

the microwave magnetic field. To decrease the noises caused by magnetic inhomogeneities on the edges of the TMF, the dimensions of the film must be a little bigger than the diameter of the measuring hole.

The operation of the detector is based on a measuring the reversal magnetization field on the TMF, which is directed along the easy magnetization axis. In the absence of a ferromagnetic sample this field will obviously coincide with the coercive force $H_{C}$ of the magnetic film area that which closes the measuring hole. As the detector we used a permalloy film with a thickness of $\sim 0.1$ microns with $H_{C}<1$ Oe, which had magnetization reversal on the measuring hole area by a single Barkhausen jump. The inverse of the magnetic moment vector associated with film magnetization reversal gives a jump change of the FMR signal polarity in the field $H_{C}$ (Fig. 2). Thus, $H_{C}$ is determined by the position of the signal jump in the recorded spectrum. The scanning spectrometer of the ferromagnetic resonance can measure $H_{C}$ with high precision, 0.01 Oe [6], due to the digital sweep of the constant magnetic field and to the lock-in detection of a signal on the modulating magnetic field frequency. It is very important that the low-frequency modulating magnetic field with frequency 
$1 \mathrm{kHz}$ present in the spectrometer acts as "a magnetic shake-up”, due to which the recurrence of good results for repeated measurements is ensured.

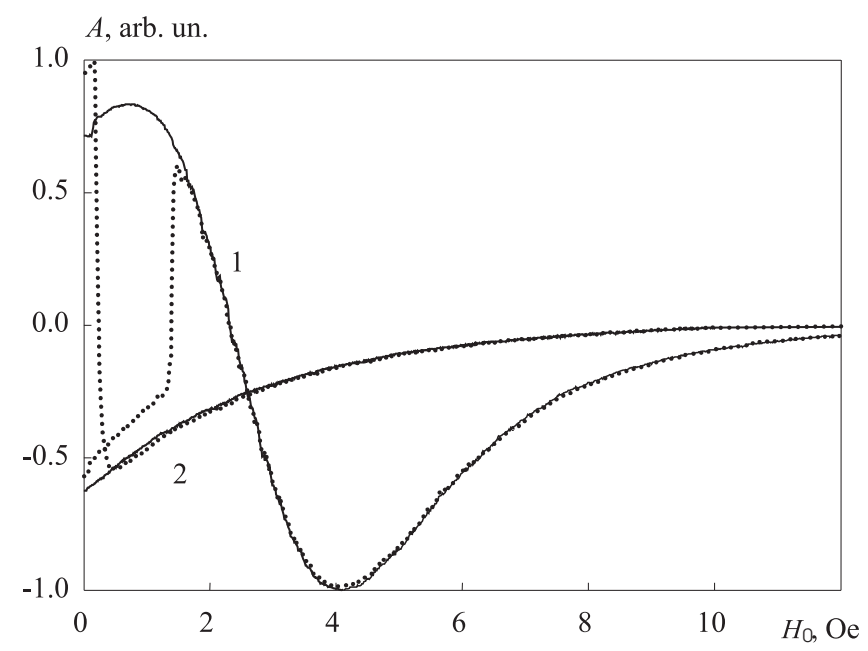

Fig. 2.: FMR curves of permalloy films having different fields of uniaxial magnetic anisotropy.

1) $H_{k}=1.5 \mathrm{Oe}$, 2) $H_{k}=4.5 \mathrm{Oe}$. Solid curves correspond to the sweeping magnetic field along the initial magnetizing, dots are the opposite sweeping.

The signal connected to the film magnetization reversal depends essentially on the microwave pumping frequency of the detector [6], and at the fixed pumping frequency it depends on the magnetic parameters of the film, and also on the anisotropy field quantity. The curves of the FMR of two permalloy films with different uniaxial magnetic anisotropy and coercive force are shown in Fig. 2. The frequency pumping was equal to $721 \mathrm{MHz}$. As research has shown [7], the greatest signals are observed when measured field $H_{C}$ falls in the medial part of the ferromagnetic resonance curve slopes of the TMF.

Optimum pumping frequencies are easy to determine from the equation:

$$
\omega_{1,2}=\gamma \sqrt{4 \pi M_{s}\left|H_{k}+H_{C} \pm \Delta H\right|}
$$

Where $\omega$ - circular frequency of MW pumping; $\gamma$ - gyromagnetic ratio; $M_{s}$ - saturation magnetization; $H_{k}$ - anisotropy field; $\Delta H$ - ferromagnetic resonance linewidth. For permalloy films the optimum frequencies fall in the $200-1500 \mathrm{MHz}$ band.

It is natural that the investigated ferromagnetic sample strongly perturbs the field homogeneity by its own field $H_{B}$ (Fig. 3), which is directed against the homogeneous magnetizing field $H_{0}$ created by the Helmholtz coils. Therefore during the sweep of constant field $H_{0}$ the TMF reverses its magnetization in some field $H_{0}=H_{d}$ which is much more than the quantity of the coercive force of the film $H C$. However, at some point of measurement $H_{C}=H_{d}-H_{B}$. As a result, the dependence of the change in the field of TMF magnetization reversal $H_{d}$ on moving the detector above an investigated sample, for example, along the line shown by the points in Fig. 3, reflects the relevant dependence of stray field $H_{B}$, enlarged on value $H_{C}$. Taking into account that $H_{C}$, as a rule,

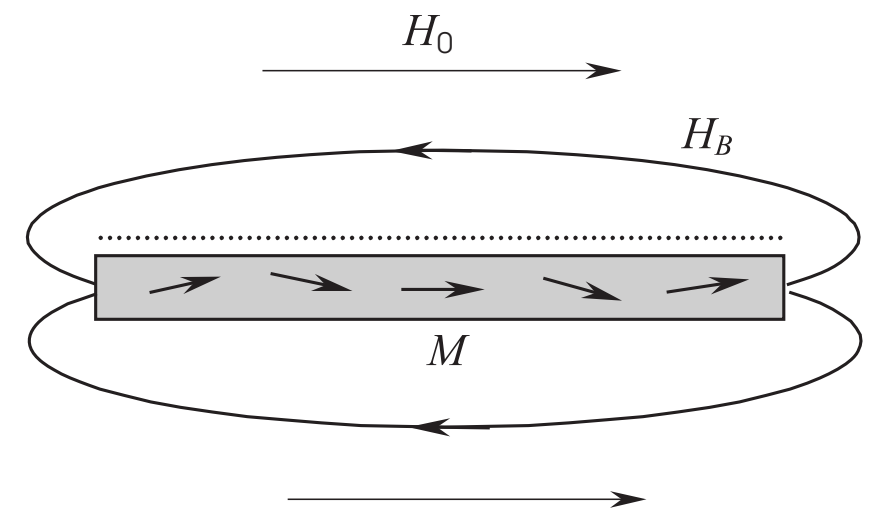

Fig. 3.: The investigated sample in a uniform magnetic field

is much lower than $H_{d}$, the magnetic film in the detector can be considered as a null-indicator showing the equality condition of the external magnetic field created by the Helmholtz coils and the tangential component of the sample stray field.

It should be noted that the magnetic film of the detector reverses the magnetization in some, averaged trough the area of the TMF, magnetic field equal to $H_{C}$ and that the film practically does not perturb the stray fields of the sample due to its small volume. With the help of the special adjusting screw of the device, the spacing between the magnetic film plane of indicator and the investigated sample surface may be regulated in a wide range between $0.02 \mathrm{~mm}$ and $5 \mathrm{~mm}$. The minimum spacing not only ensures maximum locality of measurements, but also allows the stray fields to be fixed mainly from the thin near-surface layer of the sample. As the spacing increases the thickness of the near-surface layer forming the integrated stray fields grows, and the locality of the measurements is reduced. Thus all the results shown below were obtained with minimum spacing.

\section{Experimental results}

To test the described technique for measuring stray fields, some high quality steel disks were manufactured, $48 \mathrm{~mm}$ in diameter and $4 \mathrm{~mm}$ in thickness. After manufacturing, the disks were annealed to eliminate the residual stresses in them, and then on one side of each disk half of its thickness was removed by grinding with either one-entry or two-entry. Samples manufactured in such way were placed in the centre of the stage in the FMR scanning spectrometer. [5] (see Fig. 1).

The typical behaviour of the local stray fields when the magnetizing field was increased is shown in Fig. 4. The TMF magnetization reversal field $H_{d}$ in this experiment was registered on a demagnetized sample with reverse sweeping of the magnetizing field from set value $H_{0}$ down to zero. The FMR spectra were read, starting from magnetic field $1 \mathrm{Oe}$, and after each pass the age $H_{0}$ was also incremented by 1 Oe. As a result the investigated sample was gradually more and more magnetized, and, accordingly, its stray field increased, which, as already mentioned, directed against the magnetizing field. The coercive force of the magnetic film used in the detector $H_{C}=0.15$ Oe. Therefore the occurrence of the TMF magnetization reversal signal of the detector, since field $H_{0}=21 \mathrm{Oe}$, demonstrates that up to this critical field the dif- 


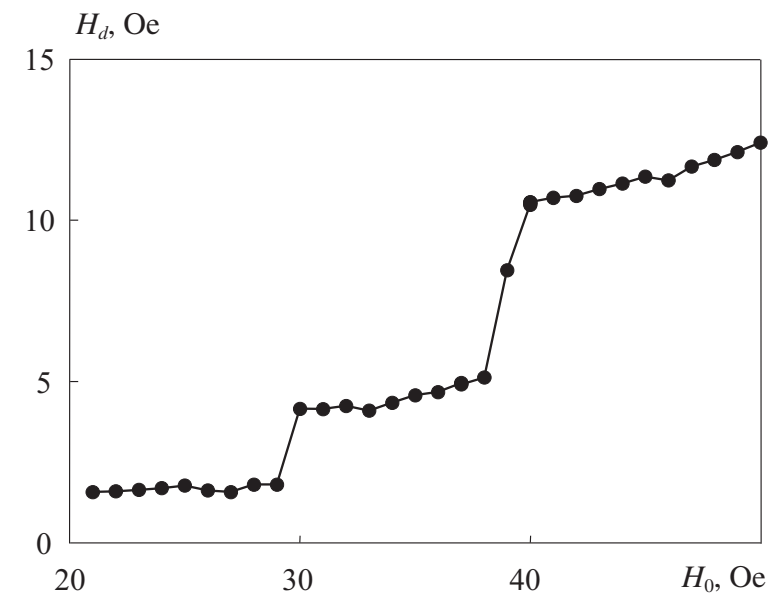

Fig. 4.: Dependence of the TMF magnetic reversal field on the value of magnetizing field applied along the direction of the sample treatment

ference between external field $H_{0}$ and sample stray field $H_{B}$ remained less than 0.15 Oe.

The apparent discrete change $H_{d}$ with growth $H_{0}$ allows us to assume that the magnetic domains in the demagnetized sample do not penetrate right through and domain structure is multi-layer. This domain structure with the growth of the magnetizing field for particular critical fields is reconstructed by jumps, similar to Barkhausen jumps, that become more and more simple. At a further increase $H_{0}$ the amplitude of these jumps is diminished, and dependence $H_{d}\left(H_{0}\right)$ gradually becomes monotonic.

Applying the experiments to many samples has shown that the number of the jumps, their amplitude $H_{d}$, and also their position depend not only on the sample material, but also on the selected local area, and mainly, on the magnetizing direction of the sample relatively to the direction of its grinding during manufacture. This demonstrates that the set of magnetization curves been recorded in local spots across the whole area of the sample contain information about the distribution of the plastic and elastic deformations formed in the sample during a mechanical treatment. Similar behaviour $H_{d}\left(H_{0}\right)$ is observed during a direct magnetic field sweep, but after first magnetizing the sample by a field that is more than critical. Depending on the strength of the magnetizing field, the value of the TMF magnetization reversal field on the direct sweep course $H_{d} \uparrow$ may be either more or less than the magnetization reversal field of the film on the reverse sweep course $H_{d} \downarrow$ (Fig. 5). This fact is explained as follows. The field $H_{d}$ measured on the direct sweep, with the increase in the magnetizing field, is saturated much earlier than the same field measured on the reverse sweep. As a result for "small" magnetizing fields the difference $\delta H_{d}=H_{d} \downarrow-H_{d} \uparrow$ is negative, while for "large" fields it is positive. And obviously there exists a quantity of the magnetizing field at which $\delta H_{d}=0$.

The observed difference of the TMF magnetization reversal fields for a direct and inverse sweep reflects the real hysteresis of the stray fields on the measured local area. The hysteresis quantity for the fixed magnetizing field depends not only on the selected local area, but also on the angle of the magnetization direction of the sample (the direction of its treatment). Therefore the hysteresis measured on local areas

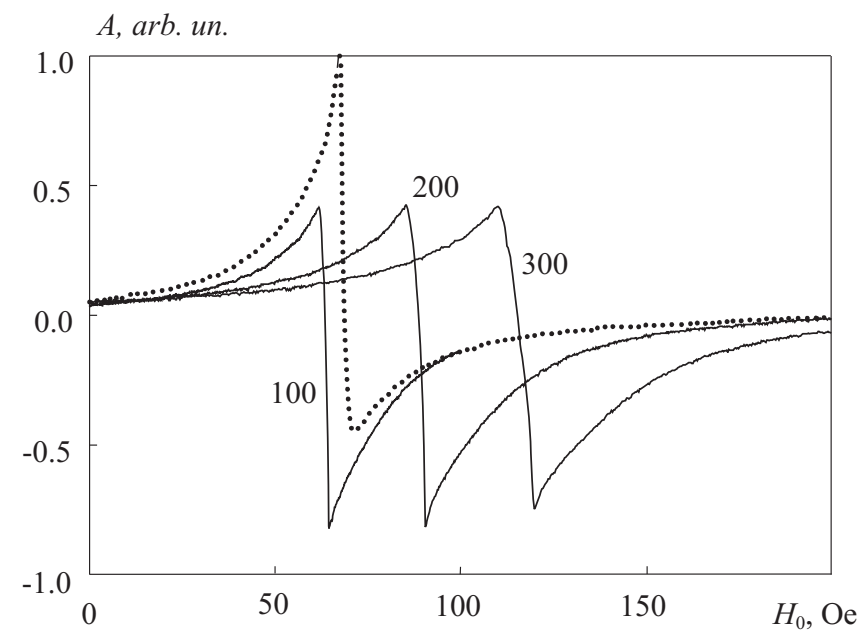

Fig. 5.: Typical FMR signals recorded with the opposite course of magnetic field sweeping from $H_{0}=100,200$ and 300 Oe. Dots display the signal obtained in the direct course of sweeping.

of the sample surface also reflects the distribution of the plastic and elastic deformations acquired by the material during its mechanical treatment. For rapid diagnostics of samples, the FMR spectrometer-controlling program provides automatic evaluation and makes a record in a file not only of the TMF magnetization reversal fields, but also of the hysteresis quantity. It is important to note that the pattern of the distribution of the inhomogeneities of the hysteresis $\delta H_{d}$ on the sample surface, and also the pattern of inhomogeneities of the magnetization reversal field $H_{d}$, depends on the quantity of the magnetizing field selected in the experiment. Using a "weak" magnetizing field $\left(H_{0}<50 \mathrm{Oe}\right)$, when $\delta H_{d}<0$, on a background of "long-wave" inhomogeneities, with a reference size of about the sample size, we can clearly observe "short-wave" inhomogeneities that are much smaller than the samples. Using "strong" fields $\left(H_{0}>100 \mathrm{Oe}\right)$ when $\delta H_{d}>0$, only the "long-wave" inhomogeneities become apparent, and the "short-wave" inhomogeneities level out. It is possible that these stray fields of "short-wave" inhomogeneities are connected to stresses reflecting the "frozen" standing waves pattern of the elastic oscillations in the sample, produced by blank vibration during manufacture.

In the patterns of „short-wave“ inhomogeneities distribution of stray fields on the disk samples, we can observe a symmetry that often has a radial structure. Fig. 6 shows the stray fields distribution for a sample manufactured by two-entry grinding along the $y$ axis. The spectra record was carried out for a reverse sweep from the field $H_{0}=20 \mathrm{Oe}$, directed along the grinding line. Note that the pattern of inhomogeneities remains as a whole the same when the sample is magnetized in the direction perpendicular to the grinding line, but the number of apparent extremes varies.

In Fig. 7 shows the stray field distribution obtained for magnetizing along the grinding line $\left(H_{d y}\right)$ and perpendicular to the grinding line $\left(H_{d x}\right)$ for a sample manufactured by one-entry grinding in direction $y$. The dependences are obtained for field reverse sweeping from $H_{0}=200 \mathrm{Oe}$, so the "short-wave" inhomogeneities show weakly on them. It can be seen that these distributions differ essentially from each 


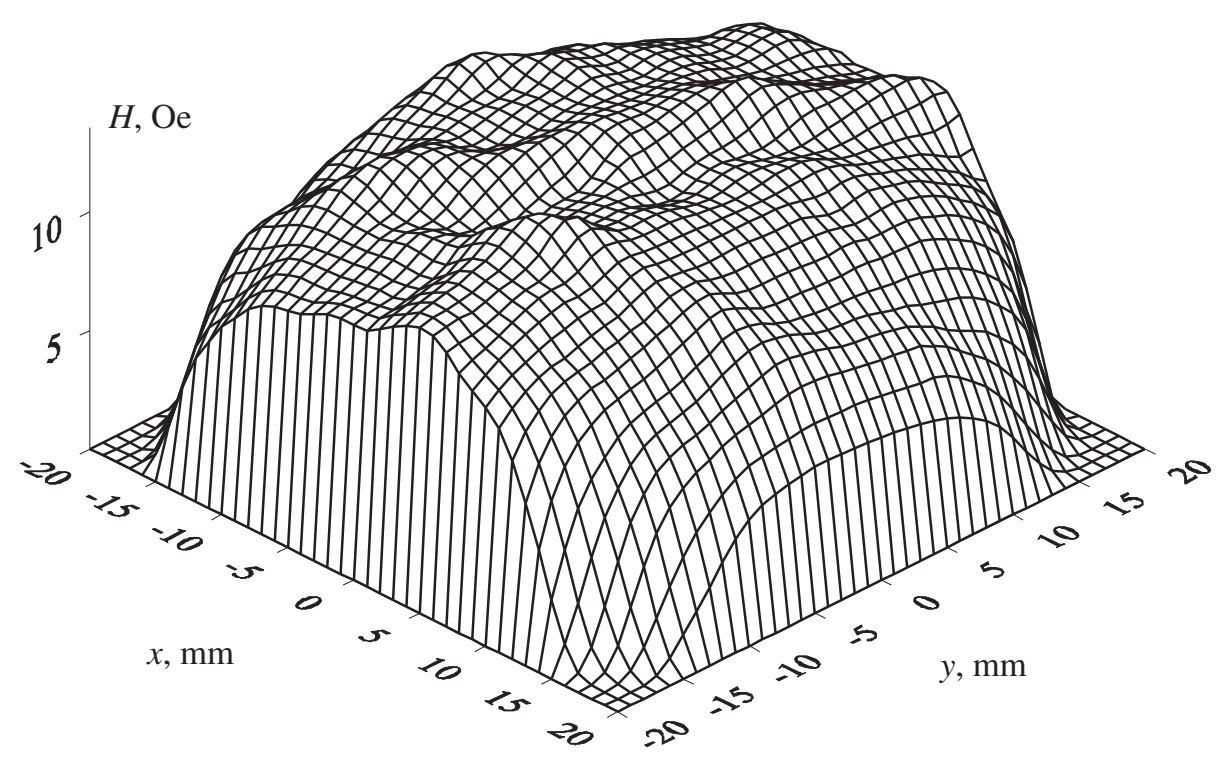

Fig. 6.: Distribution of magnetic reversal field of TMF in the detector across the surface of a sample manufactured by two-entry grinding

other, but they have good symmetry concerning the $y$ axis. Here the dependence of the difference of these fields $\Delta H_{d}=H_{d y}-H_{d x}$, where a "dent" is visible on the part where the tool enters the sample as it is being ground. A similar feature is also seen in the homogeneity hysteresis distribution $\Delta H_{d}$ (see Fig. 7). This is visible independently of the sample magnetization direction. As expected, the distribution pat-
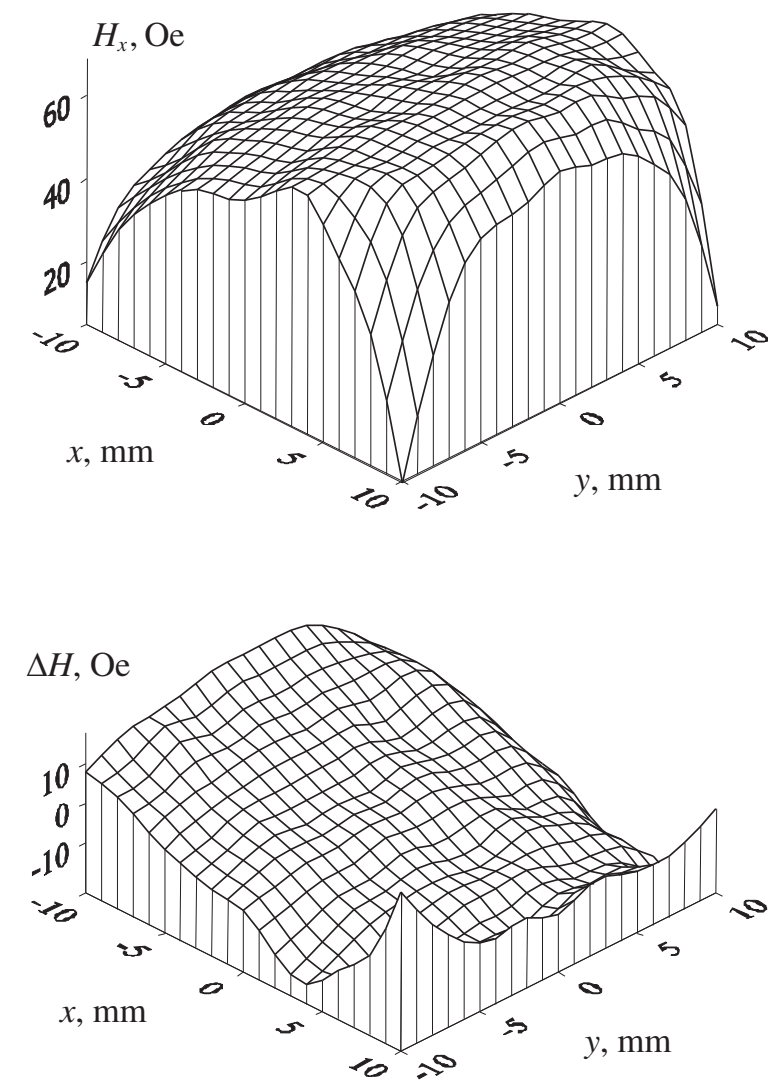

terns of the stray fields for all samples obtained by two-entry grinding have rather good symmetry not only concerning the $y$ axis, but also concerning the $x$ axis.

For "magnetic" diagnostics of cold-hardening in the ferromagnetic samples, helpful information can be received from the angular dependences of the stray fields measured for a sample previously magnetized along any chosen direction.
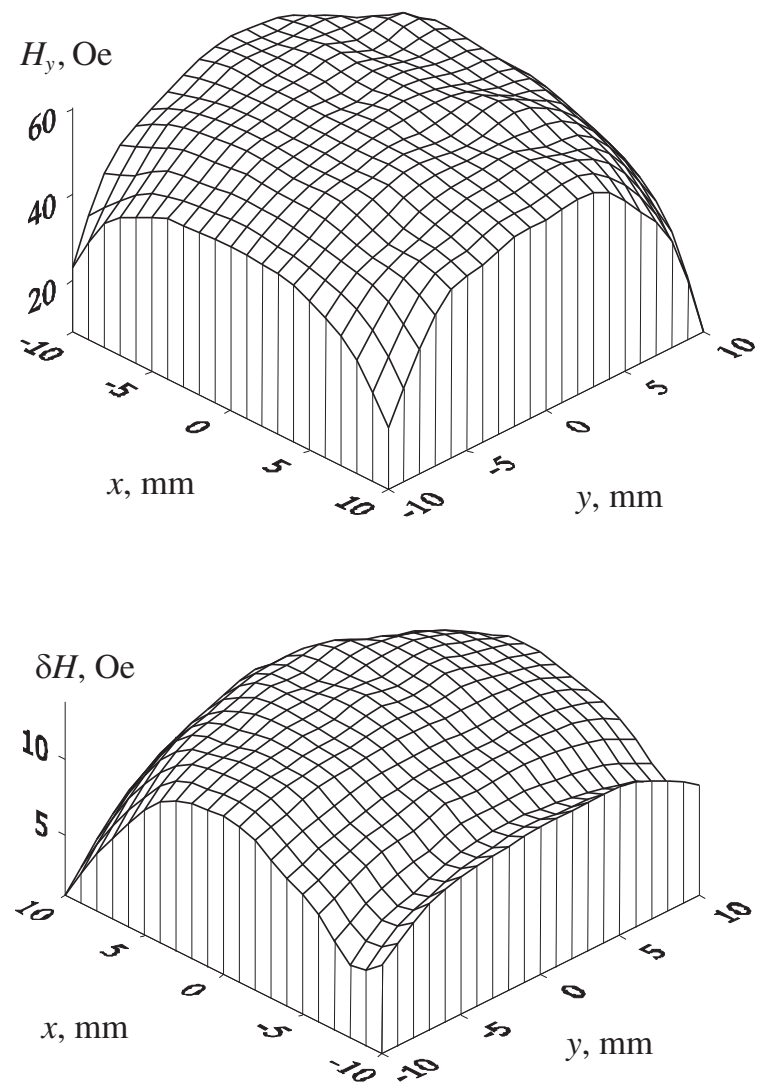

Fig. 7.: Distributions of the magnetic reversal field of TMF in the detector in the central area of a sample manufactured by one-entry grinding, $H_{d y}$ corresponds to magnetizing along the grinding direction and $H_{d x}$ corresponds to magnetizing in the perpendicular direction; $\Delta H_{d}$ is the distribution of the difference of these fields; $\delta H_{d}$ is the distribution of hysteresis. 


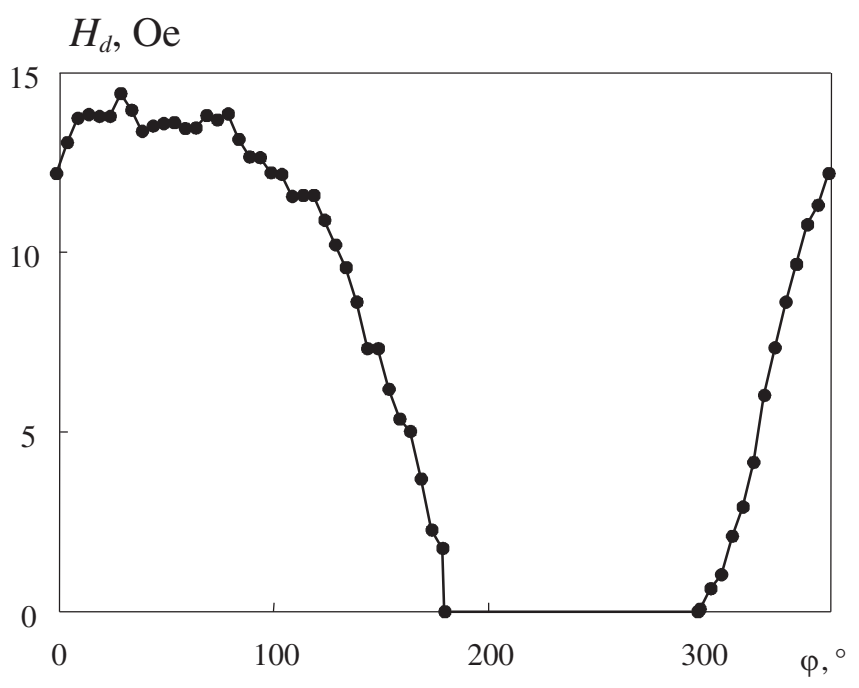

Fig. 8.: Angular dependence of the magnetic reversal field in the central area of the sample magnetized along the line of treatment

Fig. 8 shows a typical dependence of the TMF magnetization reversal field on the sweeping field direction when the detector was placed above the central segment of the sample. The sample had been magnetized in a field 100 Oe along the grinding direction. A record of spectra was made in an inverse sweep of the magnetic field from $H_{0}=20$ Oe. This field was much lower than the field in which the sample was magnetized and, as the experiment has shown, it does not in practice destroy the sample domain structure that was formed in it after its magnetization. Therefore the quantity of field Hd after rotating the sample by $360^{\circ}$ remains almost the same for the initial value at $\varphi=0^{\circ}$. Such angular dependence of the stray fields demonstrates the presence of unidirectional anisotropy in the sample. Fig. 8 shows that the region of the stray field maximum occupies a wide enough interval of angles between $0^{\circ}$ and $100^{\circ}$. This indicates that the direction of the resulting magnetic moment in the measured area of the sample strongly deviates from the initial magnetizing field direction. This is clearly due to the local magnetic anisotropy of the sample which, as already mentioned, immediately depends

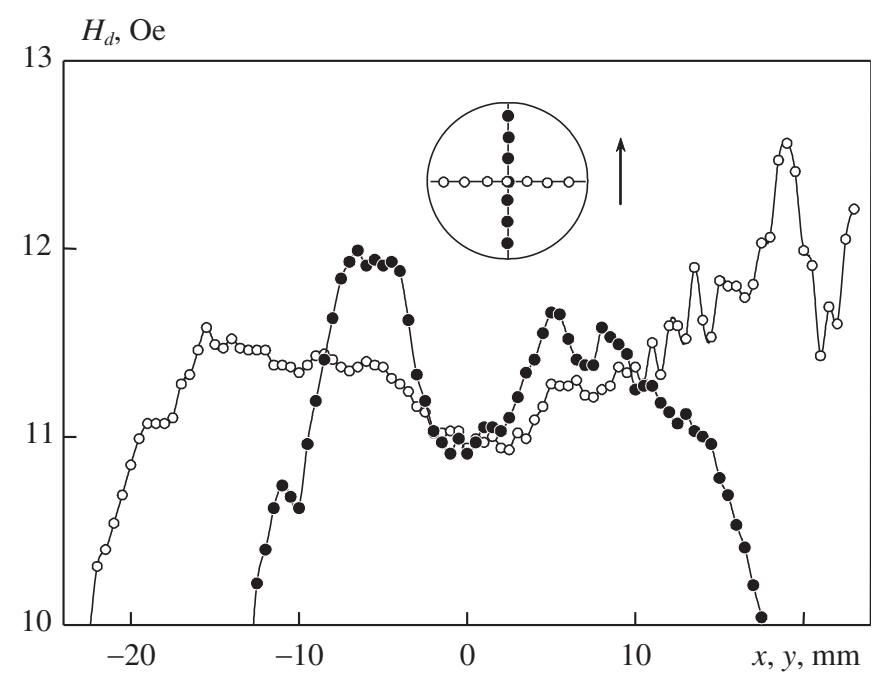

Fig. 9.: TMF magnetic reversal fields along two orthogonal directions: along the line of grinding (black points) and transversely to the line of grinding on the direction and quantity of the elastic stresses on the given local area.

Fig. 9 shows measurements of stray fields on two diameters: along (black points) and perpendicular to (white points) the direction of treatment of the sample, obtained by two-entry grinding along the $y$ axis. The dependences are measured with a step of $0.5 \mathrm{~mm}$ for a reverse sweep of the constant magnetic field from the quantity $H_{0}=20$ Oe. The constant magnetic field was directed along the line of grinding.

In this sample, measurements of the distribution of the elastic stresses were also made, using the X-ray diffraction method [8]. This method needs much time, so the measurements were carried out with larger steps of $2 \mathrm{~mm}$. The elastic stress dependences, obtained with the use of an X-ray procedure, qualitatively conform to the behaviour of the stray fields. Like $H_{d}(x), H_{d}(y)$ (see Fig. 9), they have a minimum in the centre of the sample and two maxima on each side. However, as it approaches the edges of the sample field $H_{d}$ diminished slightly faster than the apparent decrease in the elastic stresses.

It is easy to explain such a discrepancy by the influence of the "magnetic charges" existing on the sample edges, which can greatly reduce the measured field of the TMF magnetization reversal. "Magnetic charges" are especially large on the disk edges, orthogonal to the direction of the magnetization field. Therefore dependence $H_{d}$ is stronger on the $y$ axis than on the $x$ axis. In principle, the "magnetic charges" can be removed from the measurement area of the sample.

\section{Conclusion}

It is shown that cold-hardening diagnostics in ferromagnetic samples can be performed with the help of a scanning ferromagnetic resonance spectrometer. Our original measurement procedure of local stray fields with use of a thin magnetic film has high sensitivity. It allows us to record inhomogeneities of the elastic stresses arising in the sample during mechanical treatment, and in this a way to record cold-hardening indirectly. In any selected point above the sample a measurement is made of the external magnetic field, the quantity of which point compensates the sample stray fields at this point. Thus the thin magnetic film included in the measuring microwave head is a null-indicator, and the compensating point can be fixed.

Our research has also shown that the distribution of cold-hardening across the sample surface can be registered by inhomogeneities not only in the stray fields, but also by the hysteresis of these fields. In additional, the local magnetization curves and the angular dependences of stray fields can be used as signals. It is possible that in order to display the most complete and adequate pattern of plastic and elastic deformations in the sample, the whole complex of all these measurements will be needed.

The high speed and low cost of obtaining information about elastic stresses in ferromagnetic materials make our technique promising. It should be useful for making a rapid analysis improve the conditions for the manufacture of details. 


\section{References}

[1] Odintsov L. G.: Uprochnenie $i$ otdelka detaley poverkhnostnym plasticheskim deformirovaniem. Moskva, Mashinostroenie, 1987.

[2] Aseyev N. V., Dudkina N. G., Parshev S. N., Fedorov A. F.: "Metodika opredelenia velichiny uprochnenia materiala pri poverkhnostnom plasticheskom deformirovanii." Zavodskaya laboratoriya. Diagnostika materialov. Vol. 61 (1995), No. 7, p. 19.

[3] Vonsovsky S. V.: Magnetizm. Moskva, Nauka, 1971.

[4] Belyaev B. A., Leksikov A. A., Makievskii I. Ya., Ovchinnikov S. G.: Russian Patent No. 2160441.

[5] Belyaev B. A., Leksikov A. A., Makievskii I. Ya., Tyurnev V. V.: "Ferromagnetic Resonance Spectrometer." Instruments and Experimental Technique, Vol. 40 (1997), No. 3, p. 390.

[6] Belyaev B. A., Izotov A. V., Leksikov A. A.: "Skaniruyushchiy spektrometer ferromagnitnogo rezonansa dlya diagnostiki kharakteristik tonkikh magnitnykh plyonok." Zavodskaya laboratoriya. Diagnostika materialov. Vol. 67 (2001), No. 9, p. 23.

[7] Belyaev B. A., Ivanenko A. A., Leksikov A. A., Makievskii I. Ya., Pashkevich A. Z., Tyurnev V. V.: Spektrometer ferromagnitnogo rezonansa lokalnykh uchastkov tonkikh mag- nitnykh plyonok. Preprint No. 761, Institute of Physics, Krasnoyarsk, 1995.

[8] Kraus I., Ganev N.: Residual stress and stress gradients. Industrial Applications of X-Ray Diffraction. F. H. Chung, D. K. Smith (eds.). New York, Basel, 1999, p. 793.

Prof. Boris A. Belyaev

Prof. Aleksandr A. Leksikov

Sergei G. Ovchinnikov

L. V. Kirensky Institute of Physics RAS

Krasnoyarsk, Russia

Prof. RNDr. Ivo Kraus, DrSc.

phone: +420221912416

e-mail:kraus@troja.fjfi.cvut.cz

Department of Solid State Engineering

Czech Technical University in Prague

Faculty of Nuclear Sciences and Physical Engineering, Trojanova 13

12000 Praha 2, Czech Republic

Prof. Anatolyi S. Parshin

Siberian State Aerospace University

Krasnoyarsk, Russia 